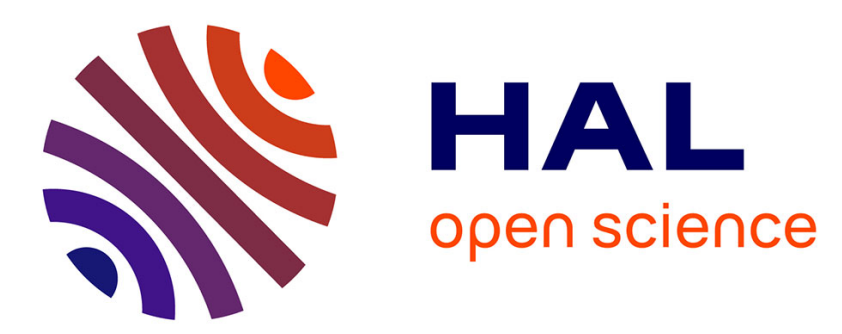

\title{
Invar, AF-Invar, Anti-Invar and Martensite in FCC Fe-Based Alloys: An Attempt to Order this Chaos
}

\author{
E. Wassermann, P. Entel
}

\section{To cite this version:}

E. Wassermann, P. Entel. Invar, AF-Invar, Anti-Invar and Martensite in FCC Fe-Based Alloys: An Attempt to Order this Chaos. Journal de Physique IV Proceedings, 1995, 05 (C8), pp.C8-287-C8-292. 10.1051/jp4:1995840 . jpa-00254089

\section{HAL Id: jpa-00254089 https://hal.science/jpa-00254089}

Submitted on 1 Jan 1995

HAL is a multi-disciplinary open access archive for the deposit and dissemination of scientific research documents, whether they are published or not. The documents may come from teaching and research institutions in France or abroad, or from public or private research centers.
L'archive ouverte pluridisciplinaire HAL, est destinée au dépôt et à la diffusion de documents scientifiques de niveau recherche, publiés ou non, émanant des établissements d'enseignement et de recherche français ou étrangers, des laboratoires publics ou privés. 


\title{
Invar, AF-Invar, Anti-Invar and Martensite in FCC Fe-Based Alloys: An Attempt to Order this Chaos
}

\author{
E.F. Wassermann and P. Entel \\ Experimentelle und Theoretische Tieftemperaturphysik und SFB 166, Gerhard-Mercator Universität, \\ 47048 Duisburg, Germany
}

\begin{abstract}
Systematics concerning magnetic and structural phase transitions within Fe-based sytems can be achieved if experimental data are analyzed as a function of composition and the atomic volume. The findings can be understood from calculations of the exchange coupling of pure fcc $\mathrm{Fe}$ as a function of the n.n. distance. Decisive is that the volume increase going along with Invar, AF-Invar, Anti-Invar as well as martensite transitions originates from a charge transfer between antibonding electronic orbitals with $t_{2 g}$ symmetry and AF exchange to non-bonding orbitals with $e_{g}$ symmetry and FM exchange. Which of the effects is leading at a given composition is a question of the position of the Fermi energy relative to these electronic levels. The question of how an effect manifests itself as a function of temperature is a question of the different strength of the $t_{2 \mathrm{~g}}$ - and $\mathrm{e}_{\mathrm{g}}$-electron-phonon coupling.
\end{abstract}

\section{FM-Invar, AF-Invar, Anti-Invar: definition of terms and relation to martensite}

In a series of recent experimental reviews [1-3] and theoretical papers [4,5] we have summarized the state of the art in the present understanding of magnetovolume effects and magnetic phase transitions in binary and ternary Fe-based alloys with fcc structure. Systems concerned are $\mathrm{Fe}$ with $3 \mathrm{~d}$-elements $(\mathrm{Cr}, \mathrm{Mn}, \mathrm{Co}$,

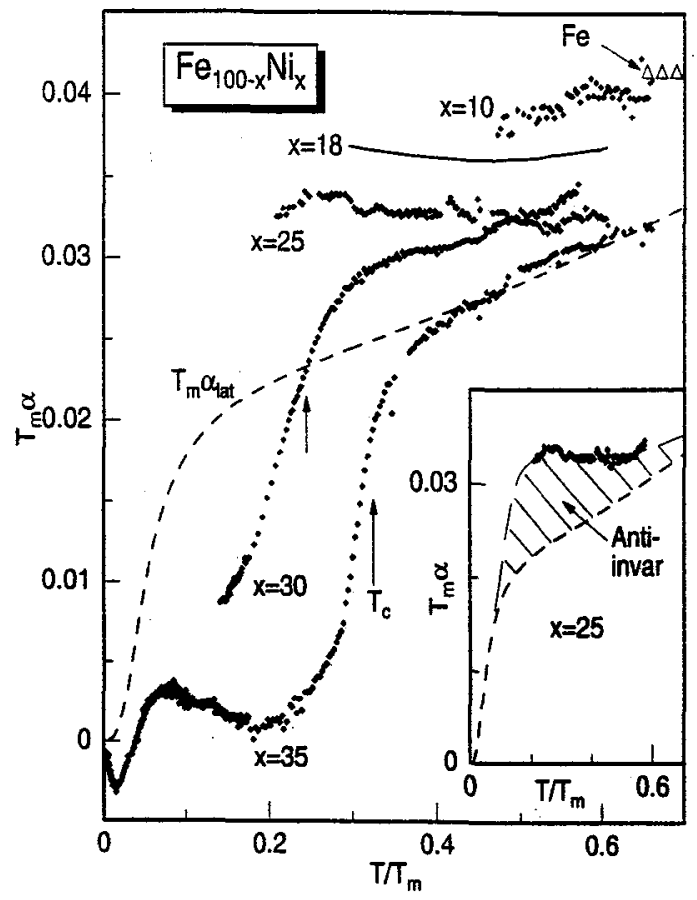

$\mathrm{Ni})$, 4d-elements (Pd) and 5d-elements (Pt, Ir).

Key words like FM-Invar for ferromagnetic (FM) alloys, AF-Invar for antiferromagnetic alloys, and Anti-Invar observed in the paramagnetic (PM) range of either FM or AF alloys, stem from the behavior of the thermal expansion coefficient $\alpha$ as a function of temperature $\mathrm{T}$. As an example we present here the well investigated FeNi system and show in Fig. 1 a plot of $\alpha$ (times the melting temperature $T_{m}$ ) versus the reduced temperature $T / T_{m}$ as measured for $\mathrm{Fe}_{100-\mathrm{x}} \mathrm{Ni}_{\mathrm{x}}$ alloys with different $\mathrm{x}$ within their fcc stability range. The reduced plot is chosen to allow for a non-magnetic (NM) reference curve $T_{m} \alpha_{\text {lat }}(T)$ which, in comparison to the experimental data clearly reveals the expansion anomalies as recently discussed [6]. For the composition $\mathrm{Fe}_{65} \mathrm{Ni}_{35}$, which has fcc stability in the whole temperature range, the "negative deviation from the reference curve in

Fig. 1 Thermal expansion coefficient $\alpha$ times the melting temperature $\mathrm{T}_{m}$ versus $\mathrm{T} / \mathrm{T}_{\mathrm{m}}$ for $\mathrm{Fe}_{\mathrm{I00-x}} \mathrm{Ni}_{x}$ alloys within the fcc stability range. 
the range below the Curie-temperature $\mathrm{T}_{\mathrm{C}}$, called FM-Invar-effect, is obvious from Fig. 1. For $\mathrm{x}=30$ at $\%$ Ni we observe besides the FM-Invar anomaly below $\mathrm{T}_{\mathrm{C}}$ a positive deviation from the reference curve in the PM range $\mathrm{T}>\mathrm{T}_{\mathrm{C}}$. This is called Anti-Invar behavior, and the hatched region shown in the inset of Fig. 1 for the alloy with $\mathrm{x}=25$ at\% Ni demonstrates, what is meant by this nomenclature. Obviously, AntiInvar behavior is found in all FeNi alloys with $\mathrm{x}<35$ at $\% \mathrm{Ni}$, even in pure fec $\gamma$-Fe in the respective stability range, as the open triangles in Fig. 1 reveal. Note that the data in Fig. 1 for the different alloys terminate at low temperatures at the respective martensite start temperatures $M_{s}$. Since AF long range ordering does not occur in the FeNi system, AF-Invar behavior in $\alpha(\mathrm{T})$ cannot directly be shown here. We remark, however, that the behavior as e.g. found in FeMn alloys [6] is similar as for FM-Invar, just the negative deviations from the reference curve below $\mathrm{T}_{\mathrm{N}}$ are generally smaller.

A systematic overview of the different physical properties of all the fcc systems with magnetovolume

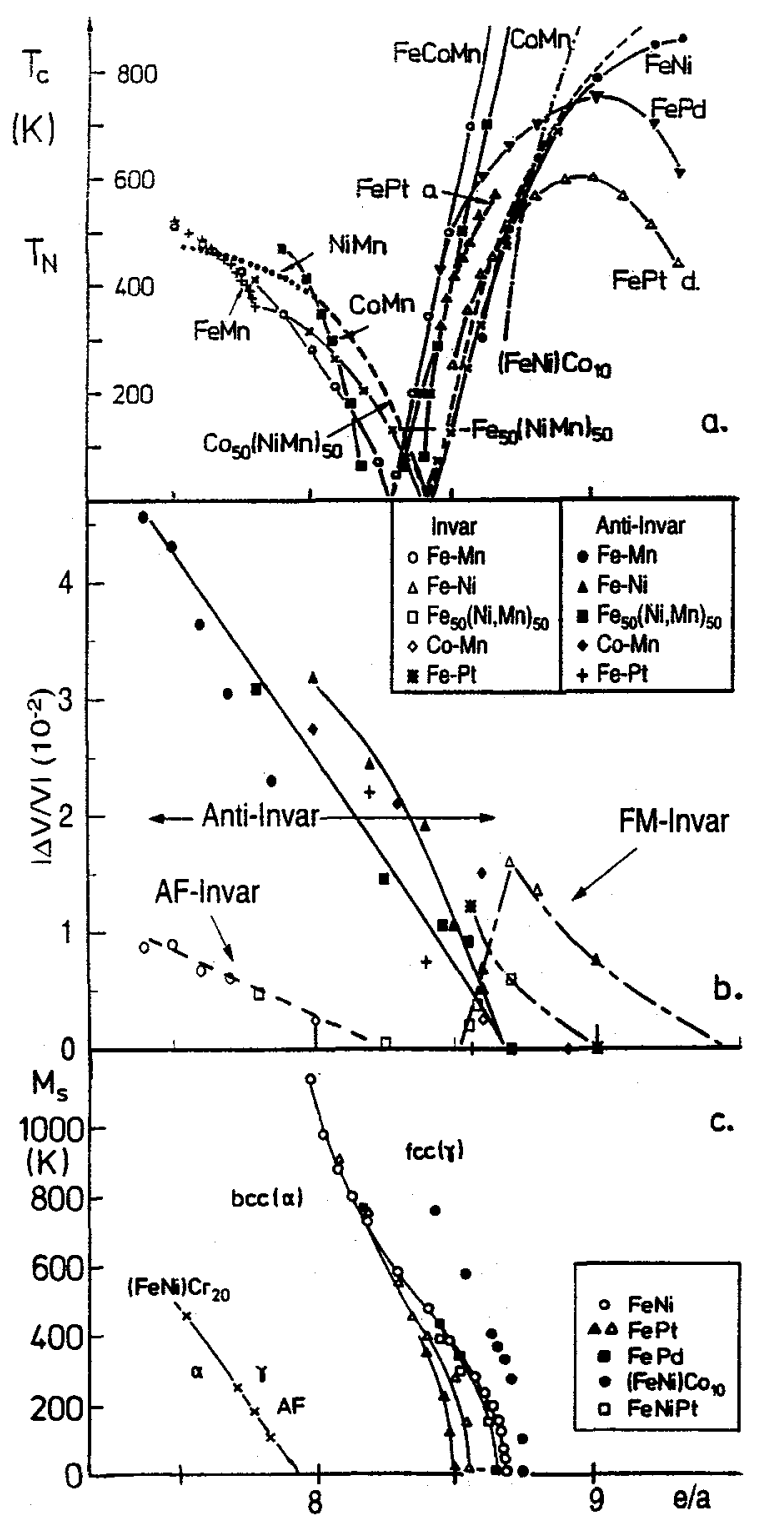
effects can be achieved, if the properties are plotted versus the composition in units of the delectron concentration per atom e/a [2]. As an example Fig. 2a shows the magnetic ordering temperatures, Curie-temperatures $T_{C}$ and Néeltemperatures $T_{N}$ versus e/a. One can see that there is an unambiguous relation between the type of magnetic ordering and e/a for all the alloys. FM order occurs only for e/a $>\sim 8.4, \mathrm{AF}$ order only for smaller electron concentrations, spin glass behavior is observed in between. This unambiguity does not exist for magnetic alloys with bcc structure. Fig. $2 b$ gives the relative volume changes associated with the different magnetovolume effects. Note that as function of e/a Anti-Invar sets in when FM-Invar dies out, the maximum volume changes associated with Anti-Invar reaching $4.5 \%$, i.e. more than twice as much than in the FM-Invar range. Note also that the AF-Invar and Anti-Invar range overlap.

A key feature with respect to the present discussion is shown in Fig. 2c, where the respective $M_{s}$ temperatures of the systems are plotted versus e/a. For $\mathrm{T}=0$ the $\gamma(\mathrm{fcc})$ to $\alpha(\mathrm{bcc})$ martensitic transition occurs right at the boundary from FM to AF order, which simultaneously is the boundary from FM-Invar to Anti-Invar, when e/a is lowered below the threshold value of $\sim 8.4$. With respect to changes in composition, FM-Invar is thus a precursor for the $\gamma \rightarrow \alpha$ martensitic transitions in the magnetic alloys, or in other words, it is

Fig.2 (a) Experimental values for Curie-temperatures $T_{c}$ and Néel-temperaturs $T_{N}$ of different ferrous alloys with fcc structure versus the d-electron concentration per atom e/a; (b) integrated, relative volume changes $\Delta V / V$ of fcc systems originating from FM- , AF-, and Anti-Invar behavior versus e/a; (c) $M_{\mathrm{s}}$ temperatures for $\gamma \rightarrow \alpha$ martensitic transitions of FM alloys and AF fcc alloys (FeNi)Cr ${ }_{20}$ versus e/a. 
the position of the Fermi energy which determines, whether FM-Invar or Anti-Invar (in some systems along with AF-Invar) together with martensite do occur. Note further that for a fixed composition at e/a $<8.4$ on lowering of the temperature, Anti-Invar is a precursor to martensite, since (like shown e.g. for FeNi in Fig. 1) Anti-Invar behavior is found at high temperatures and subsequently the martensitic transition at lower $\mathrm{T}$. This close relation between Anti-Invar and martensite calls for the same physical origin of both, as will be discussed in the following.

Before doing so we point out some differences in the martensitic transitions between non magnetic HumeRothery type systems ( $\mathrm{CuZn}, \mathrm{NiAl}, \mathrm{NiTi}$ etc.) with bcc (ordered B2) structure in the austenite phase and orthorhombic fcc type structure $(4 \mathrm{H}, 6 \mathrm{H}, 7 \mathrm{R}, 9 \mathrm{R}$ etc.) in the martensite on the one hand, and the Fe-based magnetic systems with fcc austenite and bcc (bct, fct) martensite on the other hand. The well known Hume-Rothery rule tells us that in the non-magnetic systems at $\mathrm{T}=0$ the fcc to bcc transition takes place with increasing electron concentration at $\mathrm{e} / \mathrm{a}=1.38$. For the ferrous alloys our data (Fig.2) reveal that a type of Hume-Rothery rule also holds. The fce to bcc transition here occurs around e/a $\sim 8.5$, however with decreasing e/a. A further difference between the two groups concerns the accompanying volume change. The austenite-martensite transition in the non-magnetic Hume-Rothery systems always takes place from the less dense austenite into the denser packed martensite, i.e., is accompanyed by a volume decrease. In the magnetic systems it is just the opposite, the transition is accompanyed by a volume increase. This means that the high temperature (austenitic) phase has denser package than the low temperature (martensite) phase. Reason has obviously to be searched for in the magnetic behavior of the respective systems. From the above discussion it is clear that the increase in high temperature austenite volume originates from the Anti-Invar effect (in the paramagnetic range!). It is thus necessary to understand from a microscopic standpoint the relation between magnetism and atomic volume in these systems.

\section{Origin of FM-Invar, AF-Invar, Anti-Invar and Martensite}

Basic understanding of the microscopic origin of the magnetovolume effects in question stems from band structure calculations, carried out for ordered structures by using the ASW method [4,5] and for disordered alloys with KKR-CPA [7,8]. In both cases, the total energy $E$ of a system with a pregiven lattice structure is calculated selfconsistently as a function of atomic volume $\mathrm{V}$ (or lattice constant) and the magnetic moment $M$. As examples we show in Fig.3 the total energy, relative to the minimum energy $\left(\mathrm{E}-\mathrm{E}_{0}\right)$ and the magnetic moment versus the lattice constant for bcc Fe, fcc Fe and ordered $\left(\mathrm{L1}_{2}\right) \mathrm{Fe}_{3} \mathrm{Ni}$.

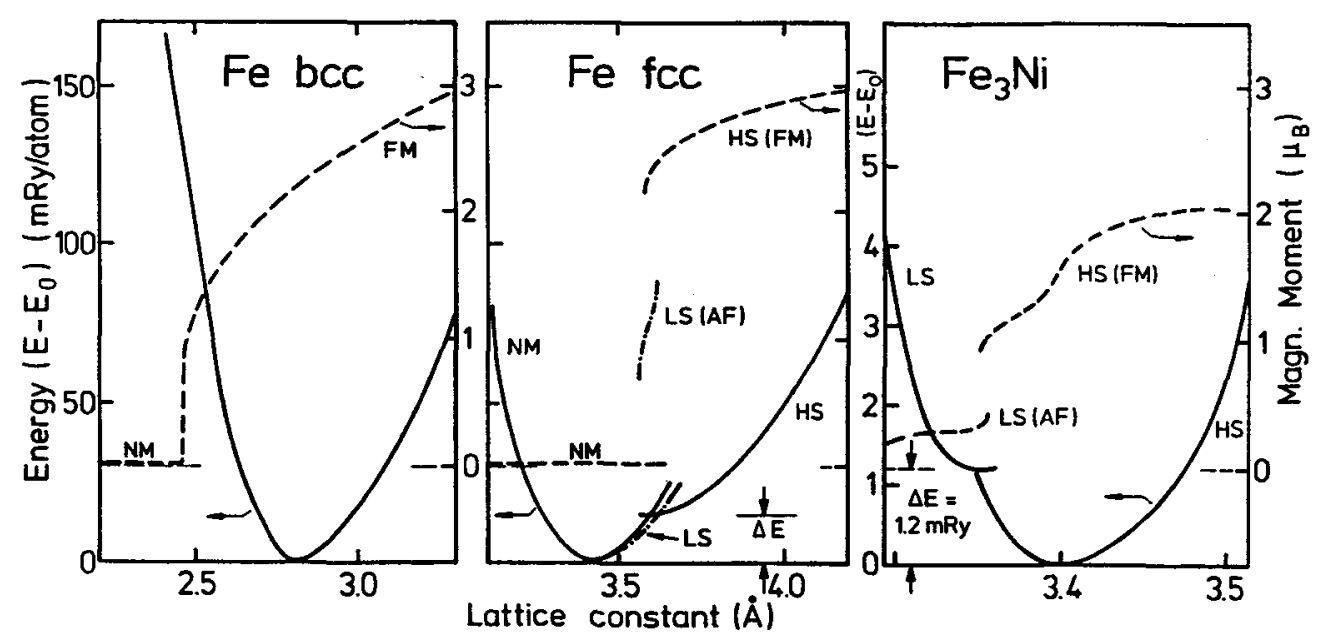

Fig. 3 Total energy (relative to a minimum energy) [left hand scales] and magnetic moment [right hand scales] versus the lattice constant for bcc and fcc $\mathrm{Fe}$ and $\mathrm{Fe}_{3} \mathrm{Ni}$ with $\mathrm{Ll}_{2}$ structure [4,5]. Note the discontinuities in the moment for fcc $\mathrm{Fe}$ and $\mathrm{Fe}{ }_{3} \mathrm{Ni}$ coupled to the occurence of so called high spin (HS) and low spin states with different magnetic order. The E(V) behavior of fcc $\mathrm{Fe}$ is typical for Anti-Invar, the $\mathrm{E}(\mathrm{V})$ curve of $\mathrm{Fe}_{3} \mathrm{Ni}$ typical for Invar alloys. 
Note that bcc Fe is a stable FM with a minimum energy at a lattice constant close to the experimental value and a magnetic moment of $2.2 \mu_{\mathrm{B}}$ at equilibrium, also in accordance with experiment. $\mathrm{M}(\mathrm{V})$ is a continuous curve which means that on application of pressure, $M$ decreases continuously, though the NM state cannot be reached in experiment. In comparison, the behavior of fcc Fe is completely different. Fcc $\mathrm{Fe}$ is obviously non-magnetic at equilibrium, but $\mathrm{E}(\mathrm{V})$ has different branches with an anharmonicity (saddle point) at increased volume. Right at this saddle point in $\mathrm{E}(\mathrm{V})$ the moment is instable (moment volume instability), so that when increasing the volume from the equilibrium value, first a small moment state with AF order, called the low spin (LS) state, and then at larger volumes the FM high spin (HS) state with large moment is reached. The HS state can indeed be realized in epitaxial fcc Fe - films on e.g. $\mathrm{Cu}_{3} \mathrm{Au}$ and manifests itself in the thermal expansion behavior in the $\gamma$ - range of fcc Fe as Anti-Invar behavior (c.f. Fig. 1). The same type of $E(V)$ curve as in fcc $\mathrm{Fe}$ is also found in the KKR-CPA calculations for disordered $\mathrm{Fe}_{100-\mathrm{x}} \mathrm{Ni}_{\mathrm{x}}$ with $\mathrm{x} \leq 32.5$ [8]. $\mathrm{E}(\mathrm{V})$ of fec $\mathrm{Fe}$ is thus typical for Anti-Invar. As Fig. 3 reveals, the reversed behavior is found in $\mathrm{Fe}_{3} \mathrm{Ni}$ Invar. Here we have the "anharmonicity" in $\mathrm{E}(\mathrm{V})$ at smaller volumes, and again a moment-volume instability at the saddle point, where a transition from a FM HS- to an AF LS-state occurs. Note that the difference $\triangle \mathrm{E}$ in energy between the HS- and the LS state is $\sim 1.2 \mathrm{mRy}$, equivalent to $\sim 200 \mathrm{~K}$. Thus, with incrasing temperature a plausible explanation for the Invar behavior results. Starting from $\mathrm{T}=0$, the normal lattice expansion is compensated by transitions from the large volume HS state to the small volumes LS state. The same type of transitions can also be induced under pressure, as Mössbauer measurements at $4.2 \mathrm{~K}$ have shown. [9]. Since the KKR-CPA calculations for disordered $\mathrm{Fe}_{100-\mathrm{x}} \mathrm{Ni}_{\mathrm{x}}$ for $\mathrm{x} \geq 35$ show similar $\mathrm{E}(\mathrm{V}$ ) curves ( $\triangle \mathrm{E}$ varying with $\mathrm{x}$ ) we can thus state that $\mathrm{E}(\mathrm{V})$ of $\mathrm{Fe}_{3} \mathrm{Ni}$ is typical for $\mathrm{FM}-$ Invar.

The understanding of the crossover from FM-Invar to Anti-Invar in fcc $\mathrm{Fe}_{100-\mathrm{x}} \mathrm{Ni}_{\mathrm{x}}$ with decreasing $\mathrm{x}$ can be deepened to a microscopic level from the band calculations, too. For FM-Invar $\mathrm{Fe}_{65} \mathrm{Ni}_{35}$ we find at the Fermi level $E_{F}$ in the respective density of states (DOS) curves energetically close lying majority-spin states of antibonding $t_{2 g}$ symmetry and minority-spin states of nonbonding $e_{g}$ symmetry. Increasing the temperature from $T=0$, there is thus the possibility of thermal excitations from these antibonding states into the nonbonding states. This can cause the volume to shrink, thus counterbalancing the normal thermal expansion arising from multi-phonon scattering. Calculations show that these electronic transitions may couple resonantly to the lattice vibrations. This would open a pseudo-gap in the electron spectrum, thereby pinning $\mathrm{E}_{\mathrm{F}}$ and hindering thermal expansion over a considerable temperature range $[5,8]$. On the other hand, increase of the $\mathrm{Fe}$ concentration (reduction of e/a) and transition into the Anti-Invar range in the FeNi system causes the Fermi level to approach the huge peak (lying below $E_{F}$ in the Invar range) in the majority DOS. In this DOS peak we find states with mainly $t_{2 g}$ symmetry which couple antiferromagnetically [10]. A very unstable situation now results, since we have coexisting FM and AF exchange interactions of nearly equal amplitude, giving rise to large spin fluctuations at high temperatures (AntiInvar effect) and to itinerant spin-glass behavior at low temperatures. For concentrations $\mathrm{x}<30$ at $\% \mathrm{Ni}$ nature avoids this unstable situation through a structural phase transition, i.e. the formation of martensite.

Although we have from theoretical side so far discussed only zero-temperature results (extensions to finite temperatures are under current investigation), we think that these results contain most of the essential physics necessary to understand (i) the HS-LS transition in the Invar region from $\mathrm{T}=0$ to finite $\mathrm{T}$ (or under pressure at $\mathbf{T}=$ const.), as well as (ii) the Anti-Invar-effekt on the Fe-rich side through the large spin fluctuations at high temperatures, stabilizing the fcc structure, until - on lowering of the temperature the martensite start temperature $M_{s}$ is reached. Below $M_{s}$ the amplitudes of the spin fluctuations become too small to stabilize the fcc structure and it is energetically more favourable to form the bcc structure. The Anti-Invar effect can thus be understood also as a kind of HS to LS transition, but with decrasing temperature, i.e. a reversed Invar behavior in the paramagnetic range.

To demonstrate some of these features Fig.4 shows for comparison the magnetic (Fig. 4a) and the martensitic (Fig. 4b) binding surface, respectively for $\mathrm{Fe}_{65} \mathrm{Ni}_{35}$ as obtained from the KKR-CPA calculations. In Fig. 4a constant energy contours (at $0.5 \mathrm{mRy}$ intervals) are projected into the M-V-plane. 
Also shown are the $\mathrm{H}=0$ and $\mathrm{P}=0$ lines, demonstrating the magnetovolume instability. Under external pressure or increasing temperature the system moves from the HS state minimum to lower volumes along
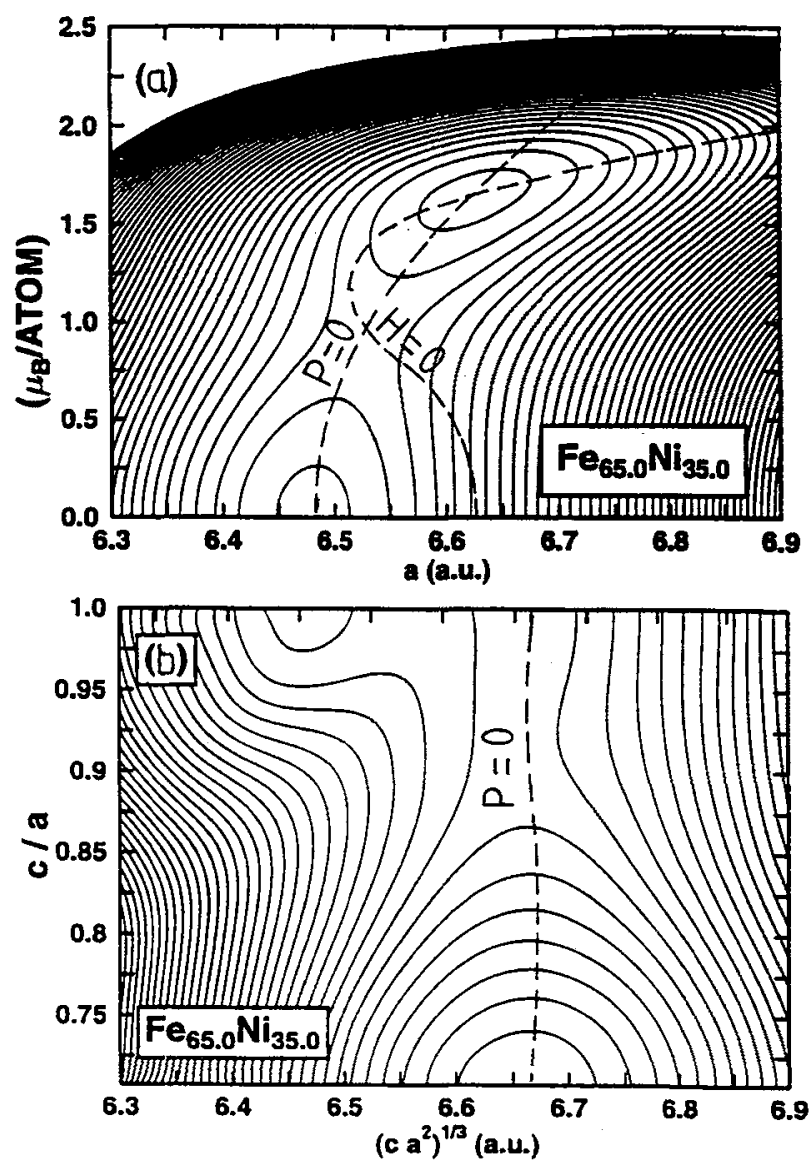

the line $\mathrm{H}=(\delta \mathrm{E} / \delta \mathrm{M})_{\mathrm{V}}=0$ until it reaches the first inflection point, where it "jumps" in a first order transition from the HS through the LS to the NM state. Fig. 4b on the other hand demonstrates the structural, first order martensitic transition along the Bain path from the fcc state at $c / a=1$ to the bcc state at $\mathrm{c} / \mathrm{a}=1 / \sqrt{2}$. Constant energy contours at $0.5 \mathrm{mRy}$ intervalls, projected into the c/a volume plane are shown. Note that $\mathbf{H}=0$ holds every where on this surface. The calculated stable ground state at this composition, lying $3 \mathrm{mRy} \sim 450 \mathrm{~K}$ below the fcc state is in contrast to the experimental result - the bcc state with FM order. However, if $\mathrm{M}=0$ is a constraint for the calculation, the fcc FM state is found to be the ground state (c.f. Fig. 14 in Ref. [8]). In Fig. $4 b$ the fcc to bcc transition occurs along $P=-(\delta E / \delta V)_{M}=0$, i.e. along a strain-free path. Along this path the elastic shear constant $C^{\prime}$ approaches zero and takes on negative values, causing the fcc

Fig. 4. (a) Magnetic and (b) martensitic binding surfaces as obtained from KKR-CPA calculations. Contour lines are in $0.5 \mathrm{mRy}$ intervalls. Ground state in (a) corresponds to $a=6.653$ a.u. and $M=1.5 \mu_{B}$ (HS state). $\mathrm{H}=\mathrm{P}=0$ lines show the moment volume instability, as the system moves under external pressure along $\mathrm{H}=0$. (b) shows how the system develops along the Bain path for $\mathrm{P}=0$, i.e. strain-free from the fcc state at $c / a=1$ to $b c c$ at $c / a=1 / \sqrt{2}$. Note that the bcc state, $3 \mathrm{mRy}$ below the fcc state is stable here, in contrast to the experimental findings.

phase to collapse spontaneously into the bcc phase. By comparing the energetic scenarios in both Fig. $4 \mathrm{a}$ and $4 \mathrm{~b}$ one can see that magnetic and structural exitation energies are of equal importance. This supports the experimental findings [1-3] that the Invar - effect is indeed a " precursor" of the martensitic transition. Actually, preliminary results show that this holds for the system FePt equally well and thus is a general feature of the fcc Fe-based magnetic alloys.

\section{Experimental and theoretical phase diagrams of $\mathrm{FeNi}$ : a comparison}

The recent theoretical findings [8], partially presented in this paper allow - though being valid at zero temperature - to get an impression of the magnetic and structural phase diagram for the FeNi system, whch can be compared to the experimental one. The results are shown in Fig. 5. Fig. 5(a) gives the experimental data, Curie-temperatures $\mathrm{T}_{\mathrm{c}}$ in the FM range and Néel-temperatures $\mathrm{T}_{\mathrm{N}}$ for "hypothetical" fcc alloys with AF order in a range, where bcc is commonly stable, hatched area means mixed magnetic ordering (FM plus AF; spin-glass like), average magnetic moment in the fcc $\left(\mathbf{M}_{\gamma}\right)$ and bcc $\left(\mathbf{M}_{\alpha}\right)$ range, and martensite transition temperature $M_{s}, M_{f}$ as well as austenite temperatures $A_{s}$ and $A_{f}$. These data can be compared to the theoretical findings, shown in Fig. $5 \mathrm{~b}$ in the following way. Comparison of the magnetic moments (open circles fcc, crosses bcc) is straight foreward. Experiment and theory agree well, though the deviation from the Slater-Pauling curve is not observed theoretically. We have shown, how- 
ever, that this deviation is of structural origin anyhow and originates from decomposition [11]. The zero temperature energy differences of the HS (or high moment $\mathrm{HM}$ ) and LS or NM states, $\mathrm{E}_{\mathrm{NM}}-\mathrm{E}_{\mathrm{HM}}$ versus composition mimic in a convincing fashion the temperature dependence of the Curie-temperatures found
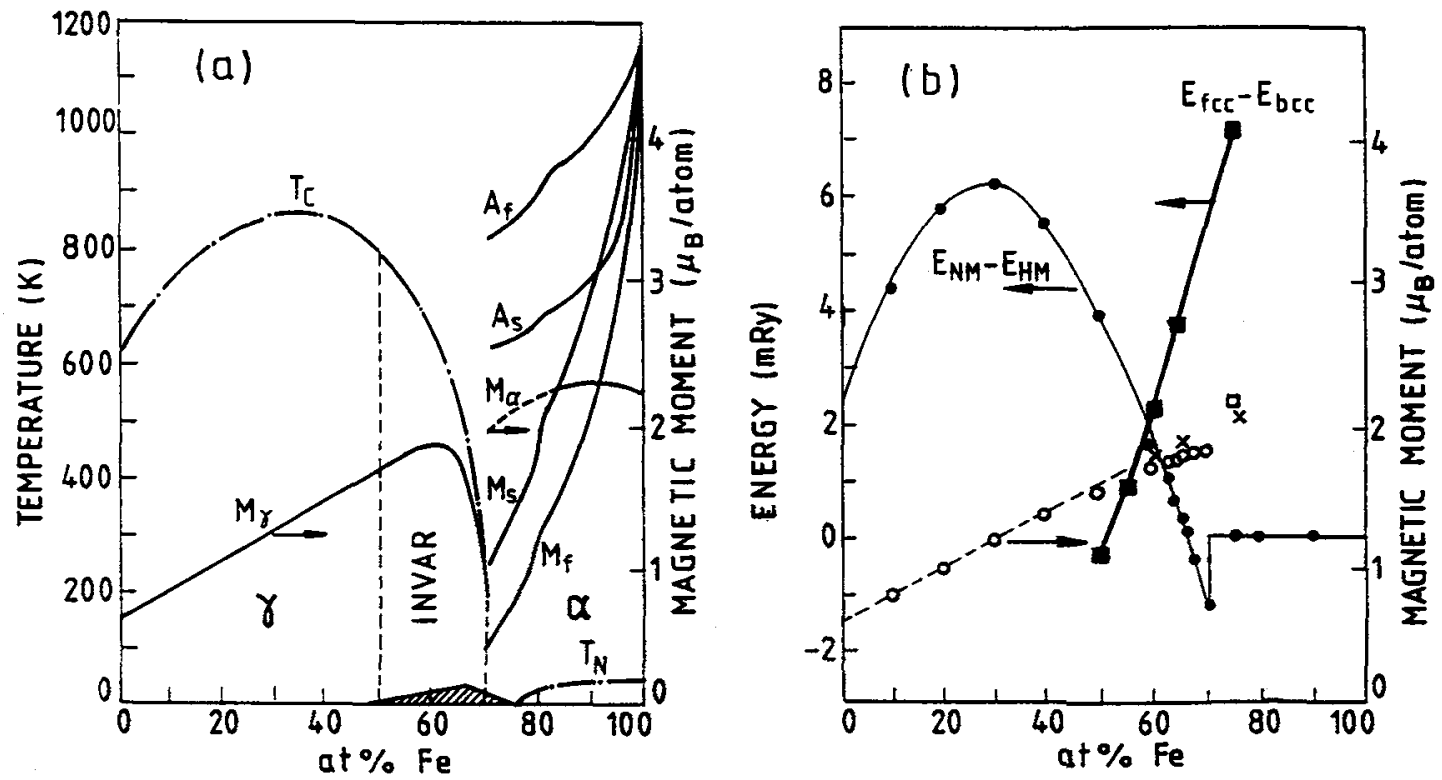

Fig.5. (a) Experimentally determined [2] magnetic phase diagram for FeNi including martensitic transition temperatures. (b) Theoretically determined magnetic phase diagram for FeNi including martensitic transition line. For details see text.

experimentally in the fcc range. Most surprising is the observation that the theoretical energy difference $\mathrm{E}_{\mathrm{fcc}}-\mathrm{E}_{\mathrm{bcc}}$ follow the average martensitic transition tempereratures $\mathrm{T}_{0}$, if these are assumed to lie half way between the respective experimental $\mathrm{M}_{\mathrm{s}}, \mathrm{M}_{\mathrm{f}}$ and $\mathrm{A}_{\mathrm{s}}, \mathrm{A}_{\mathrm{f}}$. Though the theoretical martensite transition line is shifted somewhat to lower Fe concentration as compared to the experiments, it is to our knowledge for the first time, that a martensitic transition line for a magnetic system has been calculated from first principles. In summary we may therefore say that zero-temperature first principles calculations have shown, how FM-Invar, AF-Invar, Anti-Invar and martensite are interrelated. We expect that a similar scenario will also hold for other transition metal alloy systems.

\section{References}

[1] Wassermann E.F., Physica Scripta 25 (1989) 209.

[2] Wassermann E.F.,Ferromagnetic Materials Vol.V (Buschow K.H.J. and Wohlfarth E.P., Elsevier, Amsterdam, 1990) p. 237.

[3] Wassermann E.F., J. Magn. Magn. Mat. 100 (1991) 346.

[4] Mohn P., Schwarz K., and Wagner D., Phys. Rev. B43 (1991) 3318.

[5] Entel P., Hoffmann E., Mohn P., Schwarz K., and Moruzzi V. L., Phys. Rev. B47 (1993) 8706.

[6] Schneider T., Acet M., Rellinghaus B., Wassermann E.F., and Pepperhoff W., Phys. Rev. B51 (1995) 8917.

[7] Akai H. and Dederichs P. H., Phys. Rev. B47 (1993) 8739.

[8] Schröter M., Ebert H., Akai H., Entel P., Hoffmann E., Phys. Rev. B52 (1995) 1.July.

[9] Abd-Elmeguid M.M. and Micklitz H., Phys. Rev. B40 (1989) 7395.

[10] Sabiryanov R.F., Bose S.K., and Mryasov O.N., Phys. Rev. B51 (1995) 8958.

[11] Dumpich G., Kästner J., Kirschbaum U., Mühlbauer H., Liang J,, Lübeck Th., and Wassermann E.F., Phys. Rev. B46 (1992) 9258. 